\title{
Common fixed point and invariant approximation in hyperbolic ordered metric spaces
}

Mujahid Abbas ${ }^{1}$, Mohamed Amine Khamsi ${ }^{2,3}$ and Abdul Rahim Khan ${ }^{3^{*}}$

\author{
* Correspondence: arahim@kfupm. \\ edu.sa \\ ${ }^{3}$ Department of Mathematics and \\ Statistics, King Fahd University of \\ Petroleum and Minerals, Dhahran \\ 31261, Saudi Arabia \\ Full list of author information is \\ available at the end of the article
}

\begin{abstract}
We prove a common fixed point theorem for four mappings defined on an ordered metric space and apply it to find new common fixed point results. The existence of common fixed points is established for two or three noncommuting mappings where $T$ is either ordered S-contraction or ordered asymptotically S-nonexpansive on a nonempty ordered starshaped subset of a hyperbolic ordered metric space. As applications, related invariant approximation results are derived. Our results unify, generalize, and complement various known comparable results from the current literature.

2010 Mathematics Subject Classification:

47H09, 47H10, 47H19, 54H25.
\end{abstract}

Keywords: Hyperbolic metric space, common fixed point, Ordered uniformly $\mathrm{C}_{q^{-}}$ commuting mapping, ordered asymptotically S-nonexpansive mapping, Best approximation

\section{Introduction}

Metric fixed point theory has primary applications in functional analysis. The interplay between geometry of Banach spaces and fixed point theory has been very strong and fruitful. In particular, geometric conditions on underlying spaces play a crucial role for finding solution of metric fixed point problems. Although, it has purely metric flavor, it is still a major branch of nonlinear functional analysis with close ties to Banach space geometry, see for example [1-4] and references mentioned therein. Several results regarding existence and approximation of a fixed point of a mapping rely on convexity hypotheses and geometric properties of the Banach spaces. Recently, Khamsi and Khan [5] studied some inequalities in hyperbolic metric spaces, which lay foundation for a new mathematical field: the application of geometric theory of Banach spaces to fixed point theory. Meinardus [6] was the first to employ fixed point theorem to prove the existence of invariant approximation in Banach spaces. Subsequently, several interesting and valuable results have appeared about invariant approximations [7-9].

Existence of fixed points in ordered metric spaces was first investigated in 2004 by Ran and Reurings [10], and then by Nieto and Lopez [11].

In 2009, Dorić [12] proved some fixed point theorems for generalized $(\psi, \phi)$-weakly contractive mappings in ordered metric spaces. Recently, Radenović and Kadelburg [13] presented a result for generalized weak contractive mappings in ordered metric spaces (see also, [14,15] and references mentioned theirin). Several authors studied the

(c) 2011 Abbas et al; licensee Springer. This is an Open Access article distributed under the terms of the Creative Commons Attribution License (http://creativecommons.org/licenses/by/2.0), which permits unrestricted use, distribution, and reproduction in any medium, provided the original work is properly cited. 
problem of existence and uniqueness of a fixed point for mappings satisfying different contractive conditions (e.g., $[16-18,13,19])$. The aim of this article is to study common fixed points of (i) four mappings on an ordered metric space (ii) ordered $C_{q}$-commuting mappings in the frame work of hyperbolic ordered metric spaces. Some results on invariant approximation for these mappings are also established which in turn extend and strengthen various known results.

\section{Preliminaries}

Let $(X, d)$ be a metric space. A path joining $x \in X$ to $y \in X$ is a map $c$ from a closed interval $[0, l] \subset \mathbb{R}$ to $X$ such that $c(0)=x, c(l)=y$, and $d\left(c(t), c\left(t^{\prime}\right)\right)=\left|t-t^{\prime}\right|$ for all $t, t^{\prime}$ $\in[0, l]$. In particular, $c$ is an isometry and $d(x, y)=l$. The image of $c$ is called a metric segment joining $x$ and $y$. When it is unique the metric segment is denoted by $[x, y]$. We shall denote by $(1-\lambda) x \oplus \lambda y$ the unique point $z$ of $[x, y]$ which satisfies

$$
\mathrm{d}(x, z)=\lambda \mathrm{d}(x, y), \quad \text { and } \mathrm{d}(z, y)=(1-\lambda) \mathrm{d}(x, y) .
$$

Such metric spaces are usually called convex metric spaces (see Takahashi [20] and Khan at el. [21]). Moreover, if we have for all $p, x, y$ in $X$

$$
\mathrm{d}\left(\frac{1}{2} p \oplus \frac{1}{2} x, \frac{1}{2} p \oplus \frac{1}{2} y\right) \leq \frac{1}{2} \mathrm{~d}(x, y),
$$

then $X$ is called a hyperbolic metric space. It is easy to check that in this case for all $x, y, z, w$ in $X$ and $\lambda \in[0,1]$

$$
\mathrm{d}((1-\lambda) x \oplus \lambda y, \quad(1-\lambda) z \oplus \lambda w) \leq(1-\lambda) \mathrm{d}(x, z)+\lambda \mathrm{d}(y, w) .
$$

Obviously, normed linear spaces are hyperbolic spaces [5]. As nonlinear examples one can consider Hadamard manifolds [2], the Hilbert open unit ball equipped with the hyperbolic metric [3] and $\mathrm{CAT}(0)$ spaces [4].

Let $X$ be a hyperbolic ordered metric space. Throughout this article, we assume that $(1-\lambda) x \oplus \lambda y \leq(1-\lambda) z \oplus \lambda w$ for all $x, y, z, w$ in $X$ with $x \leq z$ and $y \leq w$. A subset $Y$ of $X$ is said to be ordered convex if $Y$ includes every metric segment joining any two of its comparable points. The set $Y$ is said to be an ordered $q$-starshaped if there exists $q$ in $Y$ such that $Y$ includes every metric segment joining any of its point comparable with $q$.

Let $Y$ be an ordered $q$-starshaped subset of $X$ and $f, g: Y \rightarrow Y$. Put,

$$
Y_{q}^{f}=\left\{y_{\lambda}: y_{\lambda}=(1-\lambda) q \oplus \lambda f x \text { and } \lambda \in[0,1], q \leq x \text { or } x \leq q\right\} .
$$

Set, for each $x$ in $X$ comparable with $q$ in $Y, d\left(g x, Y_{q}^{f}\right)=\inf _{\lambda \in[0,1]} d\left(g x, y_{\lambda}\right)$.

Definition 2.1. A selfmap $f$ on an ordered convex subset $Y$ of a hyperbolic ordered metric space $X$ is said to be affine if

$$
f((1-\lambda) x \oplus \lambda y)=(1-\lambda) f x \oplus \lambda f y
$$

for all comparable elements $x, y \in Y$, and $\lambda \in[0,1]$.

Let $f$ and $g$ be two selfmaps on $X$. A point $x \in X$ is called (1) a fixed point of $f$ if $f(x)$ $=x$; (2) coincidence point of a pair $(f, g)$ if $f x=g x$; (3) common fixed point of a pair $(f$, $g$ ) if $x=f x=g x$. If $w=f x=g x$ for some $x$ in $X$, then $w$ is called a point of coincidence 
of $f$ and $g$. A pair $(f, g)$ is said to be weakly compatible if $f$ and $g$ commute at their coincidence points.

We denote the set of fixed points of $f$ by $\operatorname{Fix}(f)$.

Definition 2.2. Let $(X, \leq)$ be an ordered set. A pair $(f, g)$ on $X$ is said:

(i) weakly increasing if for all $x \in X$, we have $f x \leq g f x$ and $g x \leq f g x$, ([22])

(ii) partially weakly increasing if $f x \leq g f x$, for all $x \in X$.

Remark 2.3. A pair $(f, g)$ is weakly increasing if and only if ordered pair $(f, g)$ and $(g, f)$ are partially weakly increasing.

Example 2.4. Let $X=[0,1]$ be endowed with usual ordering. Let $f, g: X \rightarrow X$ be defined by $f x=x^{2}$ and $g x=\sqrt{x}$. Then $f x=x^{2} \leq x=g f x$ for all $x \in X$. Thus $(f, g)$ is partially weakly increasing. But $g x=\sqrt{x} \not \leq x=f g x$ for $x \in(0,1)$. So $(g, f)$ is not partially weakly increasing.

Definition 2.5. Let $(X, \leq)$ be an ordered set. A mapping $f$ is called weak annihilator of $g$ if $f g x \leq x$ for all $x \in X$.

Example 2.6. Let $X=[0,1]$ be endowed with usual ordering. Define $f, g: X \rightarrow X$ by $f x=x^{2}$ and $g x=x^{3}$. Then $f g x=x^{6} \leq x$ for all $x \in X$. Thus $f$ is a weak annihilator of $g$.

Definition 2.7. Let $(X, \leq)$ be an ordered set. A selfmap $f$ on $X$ is called dominating map if $x \leq f x$ for each $x$ in $X$.

Example 2.8. Let $X=[0,1]$ be endowed with usual ordering. Let $f: X \rightarrow X$ be defined by $f x=x^{\frac{1}{3}}$. Then $x \leq x^{\frac{1}{3}}=f x$ for all $x \in X$. Thus $f$ is a dominating map.

Example 2.9. Let $X=[0, \infty)$ be endowed with usual ordering. Define $f: X \rightarrow X$ by

$$
f x= \begin{cases}\sqrt[n]{x} & \text { for } x \in[0,1) \\ x^{n} & \text { for } x \in[1, \infty)\end{cases}
$$

$n \in N$. Then for all $x \in X, x \leq f x$ so that $f$ is a dominating map.

Definition 2.10. Let $(X, \leq)$ be a ordered set and $f$ and $g$ be selfmaps on $X$. Then the pair $(f, g)$ is said to be order limit preserving if

$$
g x_{0} \leq f x_{0},
$$

for all sequences $\left\{x_{n}\right\}$ in $X$ with $g x_{n} \leq f x_{n}$ and $x_{n} \rightarrow x_{0}$.

Definition 2.11. Let $X$ be a hyperbolic ordered metric space, $Y$ an ordered $q$-starshaped subset of $X, f$ and $g$ be selfmaps on $X$ and $q \in \operatorname{Fix}(g)$. Then $f$ is said to be:

(1) ordered $g$-contraction if there exists $k \in(0,1)$ such that

$$
\mathrm{d}(f x, f y) \leq k \mathrm{~d}(g x, g y)
$$

for $x, y \in Y$ with $x \leq y$.

(2) ordered asymptotically $S$-nonexpansive if there exists a sequence $\left\{k_{n}\right\}, k_{n} \geq 1$, with $\lim _{n \rightarrow \infty} k_{n}=1$ such that

$$
\mathrm{d}\left(f^{n}(x), f^{n}(y)\right) \leq k_{n} \mathrm{~d}(g x, g y)
$$

for each $x, y$ in $Y$ with $x \leq y$ and each $n \in N$. If $k_{n}=1$, for all $n \in N$, then $f$ is known as ordered $g$-nonexpansive mapping. If $g=I$ (identity map), then $f$ is ordered asymptotically nonexpansive mapping; 
(3) $R$-weakly commuting if there exists a real number $R>0$ such that

$$
\mathrm{d}(f g x, g f x) \leq R \mathrm{~d}(f x, g x)
$$

for all $x$ in $Y$.

(4) ordered R-subweakly commuting [23] if there exists a real number $R>0$ such that

$$
\mathrm{d}(f g x, g f x) \leq R \mathrm{~d}\left(g x, Y_{q}^{f}\right)
$$

for all $x \in Y$.

(5) ordered uniformly R-subweakly commuting [23] if there exists a real number $R>0$ such that

$$
\mathrm{d}\left(f^{n} g x, g f^{n} x\right) \leq R \mathrm{~d}\left(g x, Y_{q}^{f^{n}}\right)
$$

for all $x \in Y$.

(6) ordered $C_{q}$-commuting [24], if $g f x=f g x$ for all $x \in C_{q}(f, g)$, where $C_{q}(f, g)=U$ $\{C(g, f k): 0 \leq k \leq 1\}$ and $f_{k} x=(1-k) q \oplus k f x$.

(7) ordered uniformly $C_{q}$-commuting, if $g f^{n} x=f^{n} g x$ for all $x \in C_{q}\left(g, f^{n}\right)$ and $n \in N$.

(8) uniformly asymptotically regular on $Y$ if, for each $\eta>0$, there exists $N(\eta)=N$ such that $\mathrm{d}\left(f^{n} x, f^{n+1} x\right)<\eta$ for all $\eta \geq N$ and all $x \in Y$.

For other related notions of noncommuting maps, we refer to [7]; in particular, here Example 2.2 and Remark 3.10(2) provide two maps which are not $C_{q}$-commuting. Also, uniformly $C_{q}$-commuting maps on $X$ are $C_{q}$-commuting and uniformly $R$-subweakly commuting maps are uniformly $C_{q}$-commuting but the converse statements do not hold, in general [23,25]. Fixed point theorems in a hyperconvex metric space (an example of a convex metric space) have been established by Khamsi [26] and Park [27].

Let $Y$ be a closed subset of an ordered metric space $X$. Let $x \in X$. Define $\mathrm{d}(x, Y)=$ $\inf \{\mathrm{d}(x, y): y \in Y, y \leq x$ or $x \leq y\}$. If there exists an element $y_{0}$ in $Y$ comparable with $x$ such that $\mathrm{d}\left(x, y_{0}\right)=\mathrm{d}(x, Y)$, then $y_{0}$ is called an ordered best approximation to $X$ out of $Y$. We denote by $P_{Y}(x)$, the set of all ordered best approximation to $x$ out of $Y$. The reader interested in the interplay of fixed points and approximation theory in normed spaces is referred to the pioneer work of Park [28] and Singh [9].

\section{Common fixed point in ordered metric spaces}

We begin with a common fixed point theorem for two pairs of partially weakly increasing functions on an ordered metric space. It may regarded as the main result of this article.

Theorem 3.1. Let $(X, \leq, d)$ be an ordered metric space. Let $f, g, S$, and $T$ be selfmaps on $X,(T, f)$ and $(S, g)$ be partially weakly increasing with $f(X) \subseteq T(X), g(X) \subseteq S(X)$, and dominating maps $f$ and $g$ be weak annihilator of $T$ and $S$, respectively. Also, for every two comparable elements $x, y \in X$,

$$
d(f x, g y) \leq h M(x, y),
$$


where

$$
M(x, y)=\max \left\{d(S x, T y), d(f x, S x), d(g y, T y), \frac{d(S x, g y)+d(f x, T y)}{2}\right\}
$$

for $h \in[0,1)$ is satisfied. If one of $f(X), g(X), S(X)$, or $T(X)$ is complete subspace of $X$, then $\{f, S\}$ and $\{g, T\}$ have unique point of coincidence in $X$ provided that for a nondecreasing sequence $\left\{x_{n}\right\}$ with $x_{n} \leq y_{n}$ for all $n$ and $y_{n} \rightarrow u$ implies $x_{n} \leq u$. Moreover, if $\{f, S\}$ and $\{g, T\}$ are weakly compatible, then $f, g, S$, and $T$ have a common fixed point.

Proof. For any arbitrary point $x_{0}$ in $X$, construct sequences $\left\{x_{n}\right\}$ and $\left\{y_{n}\right\}$ in $X$ such that

$$
y_{2 n-1}=f x_{2 n-2}=T x_{2 n-1} \leq f T x_{2 n-1}, \quad \text { and } \quad y_{2 n}=g x_{2 n-1}=S x_{2 n} \leq g S x_{2 n} .
$$

Since dominating maps $f$ and $g$ are weak annihilator of $T$ and $S$, respectively so for all $n \geq 1$,

$$
x_{2 n-2} \leq f x_{2 n-2}=T x_{2 n-1} \leq f T x_{2 n-1} \leq x_{2 n-1},
$$

and

$$
x_{2 n-1} \leq g x_{2 n-1}=S x_{2 n} \leq g S x_{2 n} \leq x_{2 n} .
$$

Thus, we have $x_{n} \leq x_{n+1}$ for all $n \geq 1$. Now (3.1) gives that.

$$
\mathrm{d}\left(y_{2 n+1}, y_{2 n+2}\right)=\mathrm{d}\left(f x_{2 n}, g x_{2 n+1}\right) \leq h M\left(x_{2 n}, x_{2 n+1}\right)
$$

for $n=1,2,3, \ldots$, where

$$
\begin{aligned}
& M\left(x_{2 n}, x_{2 n+1}\right) \\
= & \max \left\{\mathrm{d}\left(S x_{2 n}, T x_{2 n+1}\right), \mathrm{d}\left(f x_{2 n}, S x_{2 n}\right), \mathrm{d}\left(g x_{2 n+1}, T x_{2 n+1}\right),\right. \\
& \left.\frac{\mathrm{d}\left(f x_{2 n}, T x_{2 n+1}\right)+\mathrm{d}\left(g x_{2 n+1}, S x_{2 n}\right)}{2}\right\} \\
= & \max \left\{\mathrm{d}\left(y_{2 n}, y_{2 n+1}\right), \mathrm{d}\left(y_{2 n+1}, y_{2 n}\right), \mathrm{d}\left(y_{2 n+2}, y_{2 n+1}\right), \frac{\mathrm{d}\left(y_{2 n+1}, y_{2 n+1}\right)+\mathrm{d}\left(y_{2 n+2}, y_{2 n}\right)}{2}\right\} \\
= & \max \left\{\mathrm{d}\left(y_{2 n}, y_{2 n+1}\right), \mathrm{d}\left(y_{2 n+1}, y_{2 n+2}\right), \frac{\mathrm{d}\left(y_{2 n}, y_{2 n+1}\right)+\mathrm{d}\left(y_{2 n+1}, y_{2 n+2}\right)}{2}\right\} \\
= & \max \left\{\mathrm{d}\left(y_{2 n}, y_{2 n+1}\right), \mathrm{d}\left(y_{2 n+1}, y_{2 n+2}\right)\right\} .
\end{aligned}
$$

Now if $M\left(x_{2 n}, x_{2 n+1}\right)=\mathrm{d}\left(y_{2 n}, y_{2 n+1}\right)$, then $\mathrm{d}\left(y_{2 n+1}, y_{2 n+2}\right) \leq h \mathrm{~d}\left(y_{2 n}, y_{2 n+1}\right)$. And if $M\left(x_{2 n}, x_{2 n+1}\right)=\mathrm{d}\left(y_{2 n+1}, y_{2 n+2}\right)$, then $\mathrm{d}\left(y_{2 n+1}, y_{2 n+2}\right) \leq h \mathrm{~d}\left(y_{2 n+1}, y_{2 n+2}\right)$ which implies that $\mathrm{d}\left(y_{2 n+1}, y_{2 n+2}\right)=0$, and $y_{2 n+1}=y_{2 n+2}$. Hence

$$
\mathrm{d}\left(y_{n}, y_{n+1}\right) \leq h \mathrm{~d}\left(y_{n-1}, y_{n}\right) \text { for } n=3,4, \ldots
$$

Therefore

$$
\begin{aligned}
\mathrm{d}\left(y_{n}, y_{n+1}\right) & \leq h \mathrm{~d}\left(y_{n-1}, x_{n}\right) \\
& \leq h^{2} \mathrm{~d}\left(y_{n-2}, y_{n-1}\right) \leq \cdots \leq h^{n} d\left(y_{0}, y_{1}\right)
\end{aligned}
$$

for all $n \in \mathbb{N}$. Then, for $m>n$,

$$
\begin{aligned}
\mathrm{d}\left(y_{n}, y_{m}\right) & \leq \mathrm{d}\left(y_{n}, y_{n+1}\right)+\mathrm{d}\left(y_{n+1}, y_{n+2}\right)+\cdots+\mathrm{d}\left(y_{m-1}, y_{m}\right) \\
& \leq\left[h^{n}+h^{n+1}+\cdots+h^{m}\right] \mathrm{d}\left(y_{0}, y_{1}\right) \\
& \leq \frac{h^{n}}{1-h} \mathrm{~d}\left(y_{0}, y_{1}\right),
\end{aligned}
$$


and so $\mathrm{d}\left(y_{n}, y_{m}\right) \rightarrow 0$ as $n, m \rightarrow \infty$. Hence $\left\{y_{n}\right\}$ is a Cauchy sequence. Suppose that $S$ $(X)$ is complete. Then there exists $u$ in $S(X)$, such that $S x_{2 n}=y_{2 n} \rightarrow u$ as $n \rightarrow \infty$. Consequently, we can find $v$ in $X$ such that $S v=u$. Now we claim that $f v=u$. Since, $x_{2 n-2}$ $\leq x_{2 n-1} \leq g x_{2 n-1}=S x_{2-n}$ and $S x_{2 n} \rightarrow S v$. So that $x_{2 n-1} \leq S v$ and since, $S v \leq g S v$ and $g S v$ $\leq v$, implies $x_{2 n-1} \leq v$. Consider

$$
\begin{aligned}
\mathrm{d}(f v, u) & \leq \mathrm{d}\left(f v, g x_{2 n-1}\right)+\mathrm{d}\left(g x_{2 n-1}, u\right) \\
& \leq h M\left(v, x_{2 n-1}\right)+\mathrm{d}\left(g x_{2 n-1}, u\right)
\end{aligned}
$$

where

$$
\begin{gathered}
M\left(v, x_{2 n-1}\right)=\max \left\{\mathrm{d}\left(S v, T x_{2 n-1}\right), \mathrm{d}(f v, S v), \mathrm{d}\left(g x_{2 n-1}, T x_{2 n-1}\right),\right. \\
\left.\frac{\mathrm{d}\left(f v, T x_{2 n-1}\right)+\mathrm{d}\left(g x_{2 n-1}, S v\right)}{2}\right\}
\end{gathered}
$$

for all $n \in \mathbb{N}$. Now we have four cases:

If $M\left(v, x_{2 n-1}\right)=\mathrm{d}\left(S v, T x_{2 n-1}\right)$, then $\mathrm{d}(f v, u) \leq h \mathrm{~d}\left(S v, T x_{2 n-1}\right)+\mathrm{d}\left(g x_{2 n-1}, u\right) \rightarrow 0$ as $n \rightarrow$ $\infty$ implies that $f v=u$.

If $M\left(v, x_{2 n-1}\right)=\mathrm{d}(f v, S v)$, then $\mathrm{d}(f v, u) \leq h \mathrm{~d}(f v, S v)+\mathrm{d}\left(g x_{2 n-1}, u\right)$. Taking limit as $n \rightarrow$ $\infty$ we get $\mathrm{d}(f v, u) \leq h \mathrm{~d}(f v, u)$. Since $h<1$, so that $f v=u$.

If $M\left(v, x_{2 n-1}\right)=\mathrm{d}\left(g x_{2 n-1}, T x_{2 n-1}\right)$, then $\mathrm{d}(f v, u) \leq h \mathrm{~d}\left(g x_{2 n-1}, T x_{2 n-1}\right)+\mathrm{d}\left(g x_{2 n-1}, u\right) \rightarrow 0$ as $n \rightarrow \infty$ implies that $f v=u$.

$$
\begin{aligned}
& \text { If } M\left(v, x_{2 n-1}\right)=\frac{\mathrm{d}\left(f v, T x_{2 n-1}\right)+\mathrm{d}\left(g x_{2 n-1}, S v\right)}{2} \text {, then } \\
& \mathrm{d}(f v, u) \leq h \frac{\left[\mathrm{d}\left(f v, T x_{2 n-1}\right)+\mathrm{d}\left(g x_{2 n-1}, S v\right)\right]}{2}+\mathrm{d}\left(g x_{2 n-1}, u\right) .
\end{aligned}
$$

Taking limit as $n \rightarrow \infty$ we get $\mathrm{d}(f v, u) \leq \frac{h}{2} \mathrm{~d}(f v, u)$. Since $h<1$, so that $f v=u$. Therefore, in all the cases $f v=S v=u$.

Since $u \in f(X) \subset T(X)$, there exists $w \in X$ such that $T w=u$. Now we shall show that $g w=u$. As, $x_{2 n-1} \leq x_{2 n} \leq f x_{2 n}=T x_{2 n+1}$ and $T x_{2 n+1} \rightarrow T w$ and so $x_{2 n} \leq T w$. Hence, $T w$ $\leq f T w$ and $f T w \leq w$, imply $x_{2 n} \leq w$. Consider

$$
\begin{aligned}
\mathrm{d}(g w, u) & \leq \mathrm{d}\left(g w, f x_{2 n}\right)+\mathrm{d}\left(f x_{2 n}, u\right) \\
& =\mathrm{d}\left(f x_{2 n}, g w\right)+\mathrm{d}\left(f x_{2 n}, u\right) \\
& \leq h M\left(x_{2 n}, w\right)+\mathrm{d}\left(f x_{2 n}, u\right)
\end{aligned}
$$

where

$M\left(x_{2 n}, w\right)=\max \left\{\mathrm{d}\left(S x_{2 n}, T w\right), \mathrm{d}\left(f x_{2 n}, S x_{2 n}\right), \mathrm{d}(g w, T w), \frac{\mathrm{d}\left(f x_{2 n}, T w\right)+\mathrm{d}\left(g w, S x_{2 n}\right)}{2}\right\}$ for all $n \in \mathbb{N}$.

Again we have four cases:

If $M\left(x_{2 n}, w\right)=\mathrm{d}\left(S x_{2 n}, T w\right)$, then $\mathrm{d}(g w, u) \leq h \mathrm{~d}\left(S x_{2 n}, T w\right)+\mathrm{d}\left(f x_{2 n}, u\right) \rightarrow 0$ as $n \rightarrow \infty$.

If $M\left(x_{2 n}, w\right)=\mathrm{d}\left(f x_{2 n}, S x_{2 n}\right)$, then $\mathrm{d}(g w, u) \leq h \mathrm{~d}\left(f x_{2 n}, S x_{2 n}\right)+\mathrm{d}\left(f x_{2 n}, u\right) \rightarrow 0$ as $n \rightarrow \infty$.

If $M\left(x_{2 n}, w\right)=\mathrm{d}(g w, T w)$, then $\mathrm{d}(g w, u) \leq h \mathrm{~d}(g w, T w)+\mathrm{d}\left(f x_{2 n}, u\right)=h \mathrm{~d}(g w, u)+\mathrm{d}\left(f x_{2 n}\right.$,

$u)$. Taking limit as $n \rightarrow \infty$ we get $\mathrm{d}(g w, u) \leq h \mathrm{~d}(g w, u)$ which implies that $g w=u$. If

$M\left(x_{2 n}, w\right)=\frac{\mathrm{d}\left(f x_{2 n}, T w\right)+\mathrm{d}\left(g w, S x_{2 n}\right)}{2}$, then 


$$
\begin{aligned}
\mathrm{d}(g w, u) & \leq h \frac{\mathrm{d}\left(f x_{2 n}, T w\right)+\mathrm{d}\left(g w, S x_{2 n}\right)}{2}+\mathrm{d}\left(f x_{2 n}, u\right) \\
& \leq \frac{h}{2}\left[\mathrm{~d}\left(f x_{2 n}, u\right)+\mathrm{d}\left(g w, S x_{2 n}\right)\right]+\mathrm{d}\left(f x_{2 n}, u\right) .
\end{aligned}
$$

Taking limit as $n \rightarrow \infty$ we get $d(g w, u) \leq \frac{h}{2} d(g w, u)$ which implies that $g w=u$. Following the arguments similar to those given above, we obtain $g w=T w=u$. Thus $\{f, S\}$ and $\{g, T\}$ have a unique point of coincidence in $X$. Now, if $\{f, S\}$ and $\{g, T\}$ are weakly compatible, then $f u=f S v=S f v=S u=w_{1}$ (say) and $g u=g T w=T g w=T u=w 2$ (say). Now

$$
\mathrm{d}\left(w_{1}, w_{2}\right)=\mathrm{d}(f u, g u) \leq h M(u, u),
$$

where

$$
\begin{aligned}
M(u, u) & =\max \left\{\mathrm{d}(S u, T u), \mathrm{d}(f u, S u), \mathrm{d}(g u, T u), \frac{\mathrm{d}(f u, T u)+\mathrm{d}(g u, S u)}{2}\right\} \\
& =d\left(w_{1}, w_{2}\right) .
\end{aligned}
$$

Therefore $\mathrm{d}\left(w_{1}, w_{2}\right) \leq h \mathrm{~d}\left(w_{1}, w_{2}\right)$ gives $w_{1}=w_{2}$. Hence

$$
f u=g u=S u=T u \text {. }
$$

That is, $u$ is a coincidence point of $f, g, S$, and $T$. Now we shall show that $u=g u$. Since, $v \leq f v=u$,

$$
\begin{aligned}
\mathrm{d}(u, g u) & =\mathrm{d}(f v, g u) \\
& \leq h M(v, u)
\end{aligned}
$$

where

$$
\begin{aligned}
M(v, u) & =\max \left\{\mathrm{d}(S v, T u), \mathrm{d}(f v, S v), \mathrm{d}(g w, T u), \frac{\mathrm{d}(f v, T u)+\mathrm{d}(g u, S v)}{2}\right\} \\
& =\mathrm{d}(u, g u) .
\end{aligned}
$$

Thus, $\mathrm{d}(u, g u) \leq h \mathrm{~d}(u, g u)$ implies that $g u=u$. In similar way, we obtain $f u=u$. Hence, $u$ is a common fixed point of $f, g, S$, and $T$.

In the following result, we establish existence of a common fixed point for a pair of partially weakly increasing functions on an ordered metric space by using a control function $r: R^{+} \rightarrow R^{+}$.

Theorem 3.2. Let $(X, \leq, d)$ be an ordered metric space. Let $f$ and $g$ be $R$-weakly commuting selfmaps on $X,(g, f)$ be partially weakly increasing with $f(X) \subseteq g(X)$, dominating map $f$ is weak annihilator of $g$. Suppose that for every two comparable elements $x, y \in X$,

$$
d(f x, f y) \leq r(d(g x, g y)),
$$

where $r: R^{+} \rightarrow R^{+}$is a continuous function such that $r(t)<t$ for each $t>0$. If either $f$ or $g$ is continuous and one of $f(X)$ or $g(X)$ is complete subspace of $X$, then $f$ and $g$ have a common fixed point provided that for a nondecreasing sequence $\left\{x_{n}\right\}$ with $x_{n} \leq y_{n}$ for all $n$ and $y_{n} \rightarrow u$ implies $x_{n} \leq u$. 
Proof. Let $x_{0}$ be an arbitrary point in $X$. Choose a point $x_{1}$ in $X$ such that

$$
f x_{n}=g x_{n+1} \leq f g x_{n+1} .
$$

Since dominating map $f$ is weak annihilator of $g$, so that for all $n \geq 1$,

$$
x_{n} \leq f x_{n}=g x_{n+1} \leq f g x_{n+1} \leq x_{n+1} .
$$

Thus, we have $x_{n} \leq x_{n}+1$ for all $n \geq 1$. Now

$$
\begin{aligned}
\mathrm{d}\left(f x_{n}, f x_{n+1}\right) & \leq r\left(\mathrm{~d}\left(g x_{n}, g x_{n+1}\right)\right) \\
& =r\left(\mathrm{~d}\left(f x_{n-1}, f x_{n}\right)\right) \\
& <\mathrm{d}\left(f x_{n-1}, f x_{n}\right) .
\end{aligned}
$$

Thus $\left\{\mathrm{d}\left(f x_{n}, f x_{n+1}\right)\right\}$ is a decreasing sequence of positive real numbers and, therefore, tends to a limit $L$. We claim that $L=0$. For if $L>0$, the inequality

$$
\mathrm{d}\left(f x_{n}, f x_{n+1}\right) \leq r\left(\mathrm{~d}\left(f x_{n-1}, f x_{n}\right)\right)
$$

on taking limit as $n \rightarrow \infty$ and in the view of continuity of $r$ yields $L \leq r(L)<L$, a contradiction. Hence, $L=0$.

For a given $\varepsilon>0$, since $r(\varepsilon)<\varepsilon$, there is an integer $k_{0}$ such that

$$
\mathrm{d}\left(f x_{n}, f x_{n+1}\right)<\varepsilon-r(\varepsilon) \quad \forall n \geq k_{0} .
$$

For $m, n \in N$ with $m>n$, we claim that

$$
\mathrm{d}\left(f x_{n}, f x_{m}\right)<\varepsilon \quad \forall n \geq k_{0} .
$$

We prove inequality (3.3) by induction on $m$. Inequality (3.3) holds for $m=n+1$, using inequality (3.2) and the fact that $\varepsilon-r(\varepsilon)<\varepsilon$. Assume inequality (3.3) holds for $m=k$. For $m=k+1$, we have

$$
\begin{aligned}
\mathrm{d}\left(f x_{n}, f x_{m}\right) & \leq \mathrm{d}\left(f x_{n}, f x_{n+1}\right)+\mathrm{d}\left(f x_{n+1}, f x_{m}\right) \\
& <\varepsilon-r(\varepsilon)+r\left(\mathrm{~d}\left(g x_{n+1}, g x_{m}\right)\right) \\
& =\varepsilon-r(\varepsilon)+r\left(\mathrm{~d}\left(f x_{n}, f x_{m-1}\right)\right) \\
& =\varepsilon-r(\varepsilon)+r\left(\mathrm{~d}\left(f x_{n}, f x_{k}\right)\right) \\
& <\varepsilon-r(\varepsilon)+r(\varepsilon)=\varepsilon .
\end{aligned}
$$

By induction on $m$, we conclude that inequality (3.3) holds for all $m \geq n \geq k_{0}$.

So $\left\{f x_{n}\right\}$ is a Cauchy sequence. Suppose that $g(X)$ is a complete metric space. Hence $\left\{f x_{n}\right\}$ has a limit $z$ in $g(X)$. Also $g x_{n} \rightarrow z$ as $n \rightarrow \infty$.

Let us suppose that the mapping $f$ is continuous. Then $f f x_{n} \rightarrow f z$ and $f g x_{n} \rightarrow f z$. Further, since $f$ and $g$ are $R$ - weakly commuting, we have

$$
\mathrm{d}\left(f g x_{n}, g f x_{n}\right) \leq R d\left(f x_{n}, g x_{n}\right)
$$

Taking limit as $n \rightarrow \infty$, the above inequality yields $g f f x_{n} \rightarrow f z$. We now assert that $z=f z$. Otherwise, since $x_{n} \leq f x_{n}$, so we have the inequality

$$
\mathrm{d}\left(f x_{n}, f f x_{n}\right) \leq r\left(\mathrm{~d}\left(g x_{n}, g f x_{n}\right)\right)
$$

Taking limit as $n \rightarrow \infty$ gives $d(z, f z) \leq r(\mathrm{~d}(z, f z))<\mathrm{d}(z, f z)$, a contradiction.

Hence, $z=f z$. As $f(X) \subseteq g(X)$, there exists $z_{1}$ in $X$ such that $z=f z=g z_{1}$. 
Now, since $f x_{n} \leq f f x_{n}$ and $f f x n \rightarrow f z=g z_{1}$ and $g z_{1} \leq f g z_{1} \leq z_{1}$ imply $f x_{n} \leq z_{1}$. Consider,

$$
\mathrm{d}\left(f f x_{n}, f z_{1}\right) \leq r\left(\mathrm{~d}\left(g f x_{n}, g z_{1}\right)\right)<\mathrm{d}\left(g f x_{n}, g z_{1}\right) .
$$

Taking limit as $n \rightarrow \infty$ implies that $f z=f z_{1}$. This in turn implies that

$$
\mathrm{d}(f z, g z)=\mathrm{d}\left(f g z_{1}, g f z_{1}\right) \leq R \mathrm{~d}\left(f z_{1}, g z_{1}\right)=0,
$$

i.e., $z=f z=g z$. Thus $z$ is a common fixed point of $f$ and $g$. The same conclusion is found when $g$ is assumed to be continuous since continuity of $g$ implies continuity of $f$.

\section{Results in hyperbolic ordered metric spaces}

In this section, existence of common fixed points of ordered $C_{q}$-commuting and ordered uniformly $C_{q}$-commuting mappings is established in hyperbolic ordered metric spaces by utilizing the notions of ordered $S$-contractions and ordered asymptotically $S$ nonexpansive mappings.

Theorem 4.1. Let $Y$ be a nonempty closed ordered subset of a hyperbolic ordered metric space $X$. Let $T$ and $S$ be ordered $R$ - subweakly commuting selfmaps on $Y$ such that $T(Y) \subset S(Y), c l(T(Y))$ is compact, $q \in$ Fix $(S)$ and $S(Y)$ is complete and q-starshaped where each $x$ in $X$ is comparable with $q$. Let $(T, S)$ be partially weakly increasing, order limit preserving and weakly compatible pair such that dominating map $T$ is weak annihilator of $S$. If $T$ is continuous, $S$-ordered nonexpansive and $S$ is affine, then $\operatorname{Fix}(T) \cap \operatorname{Fix}(S)$ is nonempty provided that for a nondecreasing sequence $\left\{x_{n}\right\}$ with $x_{n} \rightarrow$ $u$ implies that $x_{n} \leq u$.

Proof. Define $T_{n}: Y \rightarrow Y$ by

$$
T_{n}(x)=\left(1-\lambda_{n}\right) q \oplus \lambda_{n} T x,
$$

for each $n \geq 1$, where $\lambda_{n} \in(0,1)$ with $\lim _{n \rightarrow \infty} \lambda_{n}=1$. Then $T_{n}$ is a selfmap on $Y$ for each $n \geq 1$. Since $S$ is ordered affine and $T(Y) \subset S(Y)$, therefor we obtain $T_{n}(Y) \subset S$ $(Y)$. Note that,

$$
\begin{aligned}
d\left(T_{n} S_{x}, S T_{n} x\right) & =\mathrm{d}\left(\left(1-\lambda_{n}\right) q \oplus \lambda_{n} T S x,\left(1-\lambda_{n}\right) q \oplus \lambda_{n} S T x\right) \\
& \leq\left(1-\lambda_{n}\right) \mathrm{d}(q, q)+\lambda_{n} d(T S x, S T x) \\
& =\lambda_{n} \mathrm{~d}(T S x, S T x) \\
& \leq \lambda_{n} R \mathrm{~d}\left(S x,\left(1-\lambda_{n}\right) q \oplus \lambda_{n} T x\right) \\
& =\lambda_{n} R \mathrm{~d}\left(S x, T_{n} x\right) .
\end{aligned}
$$

This implies that the pair $\left\{T_{n}, S\right\}$ is ordered $\lambda_{n} R$-weakly commuting for each $n$. Also for any two comparable elements $x$ and $y$ in $X$, we get

$$
\begin{aligned}
\mathrm{d}\left(T_{n} x, T_{n} y\right) & =\mathrm{d}\left(\left(1-\lambda_{n}\right) q \oplus \lambda_{n} T x,\left(1-\lambda_{n}\right) q \oplus \lambda_{n} T y\right) \\
& \leq \lambda_{n} \mathrm{~d}(T x, T y) \leq \lambda_{n} \mathrm{~d}(S x, S y) .
\end{aligned}
$$

Now following lines of the proof of Theorem 3.2, there exists $x_{n}$ in $Y$ such that $x_{n}$ is a common fixed point of $S$ and $T_{n}$ for each $n \geq 1$. Note that

$$
\begin{aligned}
\mathrm{d}\left(x_{n}, T x_{n}\right) & =\mathrm{d}\left(T_{n} x_{n}, T x_{n}\right)=\mathrm{d}\left(\left(1-\lambda_{n}\right) q \oplus \lambda_{n} T x_{n}, T x_{n}\right) \\
& =\left(1-\lambda_{n}\right) \mathrm{d}\left(q, T x_{n}\right) .
\end{aligned}
$$

Since $c l(T(Y))$ is compact, there exists a positive integer $M$ such that

$$
\mathrm{d}\left(x_{n}, T x_{n}\right) \leq\left(1-\lambda_{n}\right) M .
$$


The compactness of $\operatorname{cl}\left(T_{n}(Y)\right)$ implies that there exists a subsequence $\left\{x_{k}\right\}$ of $\left\{x_{n}\right\}$ such that $x_{k} \rightarrow x_{0} \in Y$ as $k \rightarrow \infty$. Now,

$$
\mathrm{d}\left(x_{0}, T x_{0}\right) \leq \mathrm{d}\left(T x_{0}, T x_{k}\right)+\mathrm{d}\left(T x_{k}, x_{k}\right)+\mathrm{d}\left(x_{k}, x_{0}\right)
$$

and continuity of $T$ give that $x_{0} \in F i x(T)$. Since, $T$ is dominating map, therefore $S x_{k}$ $\leq T S x_{k}$. As $T$ is weak annihilator of $S$ and $T$ is dominating, so $T S x_{k} \leq x_{k} \leq T x_{k}$. Thus $S x_{k} \leq T x_{k}$ and order limit preserving property of $(T, S)$ implies that $S x_{0} \leq T x_{0}=x_{0}$. Also $x_{0} \leq S x_{0}$. Consequently, $S x_{0}=T x_{0}=x_{0}$. Hence the result follows.

Theorem 4.2. Let $Y$ be a nonempty closed subset of a complete hyperbolic ordered metric space $X$ and let $T$ and $S$ be mappings on $Y$ such that $T(Y-\{u\}) \subset S(Y-\{u\})$, where $u \in F i x(S)$. Suppose that $T$ is an S-contraction and continuous. Let $(T, S)$ be partially weakly increasing, dominating maps $T$ is weak annihilator of $S$. If $T$ is continuous, and $S$ and $T$ are R-weakly commuting mappings on $Y-\{u\}$, then Fix $(T) \cap F i x(S)$ is nonempty provided that for a nondecreasing sequence $\left\{x_{n}\right\}$ with $x_{n} \leq y_{n}$ for all $n$ and $y_{n} \rightarrow u$ implies $x_{n} \leq u$.

Proof. Similar to the proof of Theorem 3.2.

Theorem 3.1 yields a common fixed point result for a pair of maps on an ordered startshaped subset $Y$ of a hyperbolic ordered metric space as follows.

Theorem 4.3. Let $Y$ be a nonempty closed $q$ - starshaped subset of a complete hyperbolic ordered metric space $X$ and let $T$ and $S$ be uniformly $C_{q^{-}}$commuting selfmapps on $Y-\{q\}$ such that $S(Y)=Y$ and $T(Y-\{q\}) \subset S(Y-\{q\})$, where $q \in \operatorname{Fix}(S)$. Let $(T, S)$ be partially weakly increasing, order limit preserving and weakly compatible pair, dominating map $T$ is weak annihilator of $S, T$ is continuous and asymptotically $S$ - nonexpansive with sequence $\left\{k_{n}\right\}$, as in Definition 2.11 (2), and $S$ is an affine mapping. For each $n \geq 1$, define a mapping $T_{n}$ on $Y$ by $T_{n} x=\left(1-\alpha_{n}\right) q \oplus \alpha_{n} T^{n} x$, where $\alpha_{n}=\frac{\lambda_{n}}{k_{n}}$ and $\left\{\lambda_{n}\right\}$ is a sequence in $(0,1)$ with $\lim _{n \rightarrow \infty} \lambda_{n}=1$. Then for each $n \in N, F\left(T_{n}\right) \cap$ Fix $(S)$ is nonempty provided that for a nondecreasing sequence $\left\{x_{n}\right\}$ with $x_{n} \leq y_{n}$ for all $n$ and $y_{n}$ $\rightarrow u$ implies $x_{n} \leq u$.

Proof. For all $x, y \in Y$, we have

$$
\begin{aligned}
& \mathrm{d}\left(T_{n}(x), T_{n}(y)\right) \\
= & \mathrm{d}\left(\left(1-\alpha_{n}\right) q \oplus \alpha_{n} T^{n} x,\left(1-\alpha_{n}\right) q \oplus \alpha_{n} T^{n} y\right) \\
\leq & \alpha_{n} \mathrm{~d}\left(T^{n}(x), T^{n}(y)\right) \leq \lambda_{n} \mathrm{~d}(S x, S y) .
\end{aligned}
$$

Moreover, since $T$ and $S$ are uniformly $C_{q}$-commuting and $S$ is affine on $Y$ with $S q=q$, for each $x \in C_{n}(S, T) \subseteq C_{q}(S, T)$, we have

$$
\begin{aligned}
S T_{n} x & =S\left(\left(1-\alpha_{n}\right) q \oplus \alpha_{n} T^{n} x\right)=\left(1-\alpha_{n}\right) q \oplus \alpha_{n} S T^{n} x \\
& =\left(1-\alpha_{n}\right) q \oplus \alpha_{n} T^{n} S x=T_{n} S x .
\end{aligned}
$$

Thus $S$ and $T_{n}$ are weakly compatible for all $n$. Now, the result follows from Theorem 3.1 .

The above theorem leads to the following result.

Theorem 4.4. Let $Y$ be a nonempty closed $q$-starshaped subset of a hyperbolic ordered metric space $X$ and let $T$ and $S$ be selmaps on $Y$ such that $S(Y)=Y$ and $T(Y$ $\{q\}) \subset S(Y-\{q\}), q \in \operatorname{Fix}(S)$. Let $(T, S)$ be partially weakly increasing, order limit preserving, $T$ is continuous, uniformly asymptotically regular, asymptotically $S$-nonexpansive 
and $S$ is an affine mapping. If $c l(Y-\{q\})$ is compact and $S$ and $T$ are uniformly $C_{q}$-commuting selfmaps on $Y-\{q\}$, then $\operatorname{Fix}(T) \cap \operatorname{Fix}(S)$ is nonempty provided that for a nondecreasing sequence $\left\{x_{n}\right\}$ with $x_{n} \leq y n$ for all $n$ and $y_{n} \rightarrow u$ implies $x_{n} \leq u$.

Proof. By Theorem 4.3, for each $n \in N, F\left(T_{n}\right) \cap F i x(S)$ is singleton in $Y$. Thus,

$$
S x_{n}=x_{n}=\left(1-\alpha_{n}\right) q \oplus \alpha_{n} T^{n} x_{n} .
$$

Also,

$$
\begin{aligned}
\mathrm{d}\left(x_{n}, T^{n} x_{n}\right) & =\mathrm{d}\left(\left(1-\alpha_{n}\right) q \oplus \alpha_{n} T^{n} x_{n}, T^{n} x_{n}\right) \\
& =\left(1-\alpha_{n}\right) \mathrm{d}\left(q, T^{n} x_{n}\right) .
\end{aligned}
$$

Since $T(Y-\{q\})$ is bounded so $\mathrm{d}\left(x_{n}, T^{n} x_{n}\right) \rightarrow 0$ as $n \rightarrow \infty$. Note that,

$$
\begin{aligned}
& \mathrm{d}\left(x_{n}, T x_{n}\right) \\
\leq & \mathrm{d}\left(x_{n}, T^{n} x_{n}\right)+\mathrm{d}\left(T^{n} x_{n}, T^{n+1} x_{n}\right)+\mathrm{d}\left(T^{n+1} x_{n}, T x_{n}\right) \\
\leq & \mathrm{d}\left(x_{n}, T^{n} x_{n}\right)+\mathrm{d}\left(T^{n} x_{n}, T^{n+1} x_{n}\right)+k_{l} d\left(S T^{n} x_{n}, S x_{n}\right) \\
\leq & \mathrm{d}\left(x_{n}, T^{n} x_{n}\right)+\mathrm{d}\left(T^{n} x_{n}, T^{n+1} x_{n}\right)+k_{l} d\left(S T^{n} x_{n}, S\left(\left(1-\alpha_{n}\right) q \oplus \alpha_{n} T^{n} x_{n}\right)\right) \\
\leq & \mathrm{d}\left(x_{n}, T^{n} x_{n}\right)+\mathrm{d}\left(T^{n} x_{n}, T^{n+1} x_{n}\right)+k_{l} d\left(S T^{n} x_{n},\left(1-\alpha_{n}\right) q \oplus \alpha_{n} S T^{n} x_{n}\right) \\
\leq & \mathrm{d}\left(x_{n}, T^{n} x_{n}\right)+\mathrm{d}\left(T^{n} x_{n}, T^{n+1} x_{n}\right)+k_{1}\left(1-\alpha_{n}\right) d\left(S T^{n} x_{n}, S q\right) \\
\leq & \mathrm{d}\left(x_{n}, T^{n} x_{n}\right)+\mathrm{d}\left(T^{n} x_{n}, T^{n+1} x_{n}\right)+k_{1}\left(1-\alpha_{n}\right) d\left(S T^{n} x_{n}, S q\right) .
\end{aligned}
$$

Consequently, $\mathrm{d}\left(x_{n}, T x_{n}\right) \rightarrow 0$, when $n \rightarrow \infty$. Since $c l(Y-\{q\})$ is compact and $Y$ is closed, therefore there exists a subsequence $\left\{x_{n_{i}}\right\}$ of $\left\{x_{n}\right\}$ such that $x_{n_{i}} \rightarrow x_{0} \in Y$ as $i \rightarrow \infty$. By the continuity of $T$, we have $T\left(x_{0}\right)=x_{0}$. Since, $T$ is dominating map, therefore $S x_{k} \leq T S x_{k}$. As $T$ is weak annihilator of $S$ and $T$ is dominating, so $T S x_{k} \leq x_{k} \leq T x_{k}$. Thus, $S x_{k} \leq T x_{k}$ and order limit preserving property of $(T, S)$ implies that $S x_{0} \leq T x_{0}=x_{0}$. Also $x_{0} \leq S x_{0}$. Consequently, $S x_{0}=T x_{0}=x_{0}$. Hence, the result follows.

As another application of Theorem 3.1, we obtain yet an other result for two maps satisfying a very general contractive condition on the set $Y$.

Theorem 4.5. Let $Y$ be a nonempty $q$-starshaped complete subset of a hyperbolic ordered metric space and $T, f$, and $g$ be selfmaps on $Y$. Suppose that $T$ is continuous, $c l(T(Y))$ is compact and $f$ and $g$ are affine and continuous and $T(Y) \subset f(Y) \cap g(Y)$. Let $(T, f)$ and $(T, g)$ be partially weakly increasing, and dominating maps $f$ and $g$ be weak annihilators of $T$. If the pairs $\{T, f\}$ and $\{T, g\}$ are $C_{q}$-commuting and satisfy for all $x, y \in Y$,

$$
\begin{aligned}
\mathrm{d}(T x, T y) \leq & \max \left\{\mathrm{d}(f x, g y), \mathrm{d}\left(f x, Y_{q}^{T}\right), \mathrm{d}\left(g y, Y_{q}^{T}\right),\right. \\
& \left.\frac{1}{2}\left[\mathrm{~d}\left(f x, Y_{q}^{T}\right)+\mathrm{d}\left(g y, Y_{q}^{T}\right)\right]\right\},
\end{aligned}
$$

then $T, f$, and $g$ have a common fixed point provided that for a nondecreasing sequence $\left\{x_{n}\right\}$ with $x_{n} \leq y_{n}$ for all $n$ and $y_{n} \rightarrow u$ implies $x_{n} \leq u$.

Proof. Define $T_{n}: Y \rightarrow Y$ by

$$
T_{n}(x)=\left(1-\lambda_{n}\right) q \oplus \lambda_{n} T x,
$$

where $\lambda_{n} \in(0,1)$ with $\lim _{n \rightarrow \infty} \lambda_{n}=1$. Then $T_{n}$ is a selfmap on $Y$ for each $n \geq 1$. Since $f$ and $g$ are affine and $T(Y) \subset f(Y) \cap g(Y)$, therefore we obtain $T_{n}(Y) \subset f(Y) \cap g(Y)$. 
Now $f$ and $T$ are $C_{q}$-commuting and $f$ is affine on $Y$ with $f q=q$, for each $x \in C_{n}(f, T)$ $\subseteq C_{q}(f, T)$, so we have

$$
\begin{aligned}
f T_{n} x & =f\left(\left(1-\lambda_{n}\right) q \oplus \lambda_{n} T x\right)=\left(1-\lambda_{n}\right) q \oplus \lambda_{n} f T x \\
& =\left(1-\lambda_{n}\right) q \oplus \lambda_{n} T f x=T_{n} f x .
\end{aligned}
$$

Thus, $f$ and $T_{n}$ are weakly compatible for all $n$. Also since $g$ and $T$ are $C_{q}$-commuting and $g$ is affine on $Y$ with $g q=q$, therefore, $g$ and $T_{n}$ are weakly compatible for all $n$. Moreover using (4.1) we have

$$
\begin{aligned}
\mathrm{d}\left(T_{n} x, T_{n} y\right) \leq & \lambda_{n} \mathrm{~d}(T x, T y) \\
\leq & \lambda_{n} \max \left\{\mathrm{d}(f x, g y), d\left(f x, Y_{q}^{T(x)}\right),\right. \\
& \left.\mathrm{d}\left(g y, Y_{q}^{T(y)}\right), \frac{1}{2}\left[\mathrm{~d}\left(f x, Y_{q}^{T(y)}\right)+\mathrm{d}\left(g y, Y_{q}^{T(x)}\right)\right]\right\} \\
\leq & \lambda_{n} \max \left\{\mathrm{d}(f x, g y), \mathrm{d}\left(f x, T_{n} x\right),\right. \\
& \left.\mathrm{d}\left(g y, T_{n} y\right), \frac{1}{2}\left[\mathrm{~d}\left(f x, T_{n} y\right)+\mathrm{d}\left(g y, T_{n} x\right)\right]\right\} .
\end{aligned}
$$

By Theorem 3.1, for each $n \geq 1$, there exists $x_{n}$ in $Y$ such that $x_{n}$ is a common fixed point of $f, g$ and $T_{n}$. The compactness of $c l(T(Y))$ implies that there exists a subsequence $\left\{T x_{k}\right\}$ of $\left\{T x_{n}\right\}$ such that $T x_{k} \rightarrow y$ as $k \rightarrow \infty$. Now, the definition of $T_{k} x_{k}$ gives that $x_{k} \rightarrow y$ and the result follows using continuity of $T, f$, and $g$.

\section{Invariant approximation}

In this section, we obtain results on best approximation as a fixed point of $R$-subweakly and uniformly $R$-subweakly commuting mappings in the setting of hyperbolic ordered metric spaces. In particular, as an application of Theorem 4.4 (respectively Theorem 4.5), we demonstrate the existence of common fixed point for one pair (respectively two pairs) of maps from the set of best approximation.

Theorem 5.1. Let $M$ be a nonempty subset of a hyperbolic ordered metric space $X, T$, and $S$ be continuous selfmaps on $X$ such that $T(\partial M \cap M) \subset M, \partial M$ stands for boundary of $M$, and $u \in \operatorname{Fix}(S) \cap \operatorname{Fix}(T)$ for some $u$ in $X$, where $u$ is comparable with all $x \in X$. Let $(T, S)$ be partially weakly increasing, order limit preserving, $T$ is uniformly asymptotically regular, asymptotically $S$-nonexpansive and $S$ is affine on $P_{M}(u)$ with $S\left(P_{M}(u)\right)=P_{M}(u), q \in \operatorname{Fix}(S)$, and $P_{M}(u)$ is $q$-starshaped. If $c l\left(P_{M}(u)\right)$ is compact, $P_{M}(u)$ is complete and $S$ and $T$ are uniformly $C_{q}$-commuting mappings on $P_{M}(u) \cup\{u\}$ satisfying $\mathrm{d}(T x, T u) \leq \mathrm{d}(S x, S u)$, then $P_{M}(u) \cap \operatorname{Fix}(T) \cap \operatorname{Fix}(S) \neq \varphi$ provided that for a nondecreasing sequence $\left\{x_{n}\right\}$ with $x_{n} \leq y_{n}$ for all $n$ and $y_{n} \rightarrow u$ implies $x_{n} \leq u$.

Proof. Let $x \in P_{M}(u)$. Then $\mathrm{d}(x, u)=\mathrm{d}(u, M)$. Note that for any $\lambda \in(0,1)$,

$$
\begin{aligned}
\mathrm{d}\left(y_{\lambda}, u\right) & =\mathrm{d}((1-\lambda) u \oplus \lambda x, u) \\
& =\lambda \mathrm{d}(x, u)<\mathrm{d}(x, u)=\mathrm{d}(u, M) .
\end{aligned}
$$

This shows that $Y_{\lambda}^{I}=\left\{y_{\lambda}: y_{\lambda}=(1-\lambda) u \oplus \lambda x\right\} \cap M=\phi$. So $x \in \partial M \cap M$ which further implies that $T x \in M$. Since $S x \in P_{M}(u), u$ is a common fixed point of $S$ and $T$, therefore by the given contractive condition, we obtain

$$
\begin{aligned}
\mathrm{d}(T x, u) & =\mathrm{d}(T x, T u) \\
& \leq \mathrm{d}(S x, S u)=\mathrm{d}(S x, u)=\mathrm{d}(u, M) .
\end{aligned}
$$


Thus, $P_{M}(u)$ is $T$-invariant. Hence,

$$
T\left(P_{M}(u)\right) \subset P_{M}(u)=S\left(P_{M}(u)\right) .
$$

Now the result follows from Theorem 4.4.

Theorem 5.2. Let $M$ be a nonempty subset of a hyperbolic ordered metric space $X$, $T, f$, and $g$ be selfmaps on $X$ such that $u$ is common fixed point of $f, g$, and $T$ and $T$ $(\partial M \cap M) \subset M$. Suppose that $f$ and $g$ are continuous and affine on $P_{M}(u), q \in \operatorname{Fix}(f)$ $\cap F i x(g)$, and $P_{M}(u)$ is $q$-starshaped with $f\left(P_{M}(u)\right)=P_{M}(u)=g\left(P_{M}(u)\right)$. Let $(T, f)$ and $(T, g)$ be partially weakly increasing, and dominating maps $f$ and $g$ be weak annihilator of $T$. Assume that the pairs $\{T, f\}$ and $\{T, g\}$ are $C_{q}$-commuting and satisfy for all $x \in$ $P_{M}(u) \cup\{u\}$

$$
\mathrm{d}(T x, T y) \leq \begin{cases}\mathrm{d}(f x, g u), & \text { if } y=u \\ \max \left\{\mathrm{d}(f x, g y), \mathrm{d}\left(f x, Y_{q}^{T}\right), \mathrm{d}\left(g y, Y_{q}^{T}\right),\right. & \text { if } y \in P_{M}(u) .\end{cases}
$$

If $c l\left(P_{M}(u)\right)$ is compact and $P_{M}(u)$ is complete, then $P_{M}(u) \cap F i x(T) \cap F i x(f) \cap$ Fix $(g)$ $\neq \varphi$ provided that for a nondecreasing sequence $\left\{x_{n}\right\}$ with $x_{n} \leq y_{n}$ for all $n$ and $y_{n} \rightarrow u$ implies $x_{n} \leq u$.

Proof. Let $x \in P_{M}(u)$. Then $\mathrm{d}(x, u)=\mathrm{d}(u, M)$. Note that for any $\lambda \in(0,1)$

$$
\begin{aligned}
\mathrm{d}\left(y_{\lambda}, u\right) & =\mathrm{d}((1-\lambda) u \oplus \lambda x, u) \\
& =\lambda \mathrm{d}(x, u)<\mathrm{d}(x, u)=\mathrm{d}(u, M),
\end{aligned}
$$

which shows that $M$ and $Y_{\lambda}^{x}=\left\{y_{\lambda}: y_{\lambda}=(1-\lambda) u \oplus \lambda x\right\}$ are disjoint. So $x \in \partial M \cap M$ which further implies that $T x \in M$. Since $f x \in P_{M}(u), u$ is a common fixed point of $f$, $g$, and $T$, therefore by the given contractive condition, we obtain

$$
\begin{aligned}
\mathrm{d}(T x, u) & =d(T x, T u) \\
& \leq \mathrm{d}(f x, g u)=\mathrm{d}(f x, u)=\mathrm{d}(u, M) .
\end{aligned}
$$

Thus $P_{M}(u)$ is $T$-invariant. Hence,

$$
T\left(P_{M}(u)\right) \subset P_{M}(u)=f\left(P_{M}(u)\right)=g\left(P_{M}(u)\right) .
$$

The result follows from Theorem 4.5.

Remark 5.3.

(a) Theorem 3.2 extends and improves Theorem 2.2 of Al-Thagafi [8] and Theorem 2.2(i) of Hussain and Jungck [25] in the setup of hyperbolic ordered metric spaces.

(b) Theorems 4.4 and 4.5 extend the results in [23] to more general classes of mappings defined on a hyperbolic ordered metric space.

(c) Theorems 5.1 and 5.2 set analogues of Theorems 2.11(i) and 2.12(i) in [25], respectively. 


\section{Authors' contributions}

The authors have contributed in this work on an equal basis. All authors have read and approved the final manuscript.

\section{Competing interests}

The authors declare that they have no competing interests.

Received: 30 January 2011 Accepted: 4 August 2011 Published: 4 August 2011

\section{References}

1. Bridson, M, Haefliger, A: Metric Spaces of Non-Positive Curvature. Springer, Berlin, Heidelberg (1999)

2. Busemann, H: Spaces with non positive curvature. Acta Math. 80, 259-310 (1984)

3. Goebel, K, Reich, S: Uniform Convexity, Hyperbolic Geometry and Gonexpansive Mappings. Marcel Dekker, Inc., New York (1984)

4. Kirk, WA: Fixed point theory for nonexpansive mappings, I and II. In Lecture Notes in Mathematics, vol. 886, pp. 485-405. Springer, Berlin (1981)

5. Khamsi, MA, Khan, AR: Inequalities in metric spaces with applications. Nonlinear Analy. 74, 4036-4045 (2011). doi:10.1016/j.na.2011.03.034

6. Meinardus, G: Invarianz bei linearn approximation. Arch. Ration. Mech. Anal. 14, 301-303 (1963)

7. Akbar, F, Khan, AR: Common fixed point and approximation results for noncommuting maps on locally convex spaces. Fixed Point Theory Appl 14 (2009). (Article ID 207503)

8. Al-Thagafi, MA: Common fixed points and best approximation. J. Approx. Theory. 85, 318-320 (1996). doi:10.1006/ jath. 1996.0045

9. Singh, SP: Application of a fixed point theorem to approximation theory. J Approx Theory. 25, 88-89 (1979)

10. Ran, ACM, Reurings, MCB: A fixed point theorem in partially ordered sets and some application to matrix equations. Proc. Amer. Math. Soc. 132, 1435-1443 (2004). doi:10.1090/S0002-9939-03-07220-4

11. Nieto, JJ, Lopez, RR: Contractive mapping theorems in partially ordered sets and applications to ordinary differential equations. Order. 22, 223-239 (2005). doi:10.1007/s11083-005-9018-5

12. Dorić, D: Common fixed point for generalized $(\psi, \varphi)$-weak contractions. Appl. Math. Lett. 22, 1896-1900 (2009). doi:10.1016/j.aml.2009.08.001

13. Radenović, S, Kadelburg, Z: Generalized weak contractions in partially ordered metric spaces. Comput. Math. Appl. 60, 1776-1783 (2010). doi:10.1016/j.camwa.2010.07.008

14. Saadati, R, Vaezpour, SM: Monotone generalized weak contractions in partially ordered metric spaces. Fixed Point Theory. 11, 375-382 (2010)

15. Saadati, R, Vaezpour, SM, Vetro, P, Rhoades, BE: Fixed point theorems in generalized partially ordered G-metric spaces Math. Comput. Model. 52, 797-801 (2010). doi:10.1016/j.mcm.2010.05.009

16. Amini-Harandi, A, Emami, H: A fixed point theorem for contraction type maps in partially ordered metric spaces and application to ordinary differential equations. Nonlinear Anal. 72, 2238-2242 (2010). doi:10.1016/j.na.2009.10.023

17. Beg, I, Abbas, M: Coincidence point and invariant approximation for mappings satisfying generalized weak contractive condition. Fixed Point Theory Appl2006, 7. (Article ID 74503)

18. Harjani, J, Sadarangani, K: Fixed point theorems for weakly contractive mappings in partially ordered sets. Nonlinear Anal. 71, 3403-3410 (2009). doi:10.1016/j.na.2009.01.240

19. Rhoades, BE: Some theorems on weakly contractive maps. Nonlinear Anal. 47, 2683-2693 (2001). doi:10.1016/S0362546X(01)00388-1

20. Takahashi, W: A convexity in metric space and nonexpansive mappings. Kodai Math. Sem. Rep. 22, 142-149 (1970). doi:10.2996/kmj/1138846111

21. Khan, AR, Fukhar-ud-din, H, Domlo, AA: Approximating fixed points of some maps in uniformly convex metric spaces. Fixed Point Theory Appl2010, 11. (Article ID 385986)

22. Altun, I, Damjanovic, B, Djoric, D: Fixed point and common fixed point theorems on ordered cone metric spaces. Appl. Math. Lett. 23: 310-316 (2010)

23. Beg, I, Sahu, DR, Diwan, SD: Approximation of fixed points of uniformly R-subweakly commuting mappings. J. Math. Anal. Appl. 324, 1105-1114 (2006). doi:10.1016/j.jmaa.2006.01.024

24. Hussain, N, Rhoades, BE: $C_{q}$-commuting maps and invariant approximations. Fixed Point Theory Appl2006, 9. (Article ID 24543)

25. Hussain, N, Jungck, G: Common fixed point and invariant approximation results for noncommuting generalized $(f, g)$ nonexpansive maps. J. Math. Anal. Appl. 321, 851-861 (2006). doi:10.1016/j.jmaa.2005.08.045

26. Khamsi, MA: KKM and Ky Fan theorems in hyperconvex metric spaces. J. Math. Anal. Appl. 204, 298-306 (1996). doi:10.1006/jmaa.1996.0438

27. Park, S: Fixed point theorems in hyperconvex metric spaces. Nonlinear Anal. 37, 467-472 (1999). doi:10.1016/S0362546X(98)00061-3

28. Park, S: Best approximations, inward sets and fixed points. Progress in Approximation Theory. pp. 711-719.Acedemic Press, Inc (1991)

doi:10.1186/1687-1812-2011-25

Cite this article as: Abbas et al: Common fixed point and invariant approximation in hyperbolic ordered metric spaces. Fixed Point Theory and Applications 2011 2011:25. 